\title{
THE EFFECT OF THE CHRONIC ADMINISTRATION OF ADRENAL MEDULLARY HORMONES TO MAN ON ADRENOCORTICAL FUNCTION AND THE RENAL EXCRETION OF ELECTROLYTES
}

\author{
BY LEROY E. DUNCAN, JR., DAVID H. SOLOMON, MARY P. NICHOLS, AND \\ ESTHER ROSENBERG
}

\author{
(From the National Heart Institute, National Institutes of Health, Bethesda, Md., and the \\ Section on Gerontology, Baltimore City Hospitals, Baltimore, Md.)
}

(Submitted for publication April 20, 1951; accepted June 18, 1951)

The acute and chronic administration of adrenaline to experimental animals and its acute administration to man have been shown to increase adrenocortical activity.

In 1908 Babes and Jonesco reported that repeated injections of adrenaline caused adrenocortical hypertrophy in rabbits (1). Vogt found by direct assay of adrenal venous blood of dogs and cats that there was an increased output of adrenocortical hormone following the injection of adrenaline (2). She subsequently reported that multiple injections of adrenaline produced adrenal hypertrophy in intact but not in hypophysectomized rats (3).

Long and Fry described a decrease in adrenocortical cholesterol and ascorbic acid following the injection of adrenaline in intact but not in hypophysectomized rats (4). This and subsequent work by Gershberg and associates (5) indicate that adrenaline causes an increased output of adrenocorticotrophin.

Using a decrease in the level of circulating eosinophils as a very sensitive measure of increased adrenocortical activity, Recant and colleagues showed that a single dose of adrenaline increases adrenocortical activity in the dog, rat, and man (6). Although they found eosinopenia in men receiving multiple injections of adrenaline over periods as long as 48 hours, there was no evidence of increased adrenocortical activity as measured by the urinary excretion of steroids and uric acid. However, Bliss, Rubin, and Gilbert (7) reported increases in urinary uric acid and 17-ketosteroids in normal men and women given $8 \mathrm{mg}$. of adrenaline in oil in a period of 12 hours.

Guest and coworkers showed that sustained eosinopenia does not occur uniformly in patients given repeated injections of adrenaline (8).
Adrenaline prepared from biological material contains about 85 per cent epinephrine and 15 per cent nor-epinephrine (9) unless a special process is included to remove nor-epinephrine. The adrenaline used in the present study was manufactured from biological material and did contain nor-epinephrine. Since nor-epinephrine produces little or no eosinopenia $(10,11)$, the increased adrenocortical function which follows the administration of adrenaline must be due primarily to its epinephrine content. However, some of the results reported in the present paper may well have been due to nor-epinephrine. The designation adrenaline is used in this communication to indicate a mixture of epinephrine and norepinephrine.

The observations reported were undertaken to determine what manifestations of increased adrenocortical activity occur during the chronic administration of adrenaline to man. To this end the level of circulating eosinophils, the urinary excretion of uric acid, the urinary excretion of steroids, and the metabolism of nitrogen, sodium, chloride, potassium, phosphorus, and calcium have been studied in men receiving repeated injections of adrenaline over a period of days.

Among the effects observed have been increased urinary excretion of sodium, chloride, calcium, and uric acid, and decreased urinary excretion of potassium. The changes in the urinary excretion of sodium, potassium, and chloride are opposite to those seen with increased adrenocortical activity. These changes in urinary excretion are of interest in connection with previous demonstrations in experimental animals that although adrenaline in large dosage causes oliguria, adrenaline in smaller amounts produces diuresis of water and chloride (12-16). 
Increased excretion of sodium and chloride and diminished excretion of potassium were observed by Bliss, Rubin and Gilbert in a patient with rheumatoid arthritis given $8 \mathrm{mg}$. of adrenaline in oil per day for three days (7).

\section{EXPERIMENTAL METHODS}

Chemical methods. The subjects lived on a metabolic ward during the study. Food was prepared in a kitchen used only for this purpose. Aliquots of diet and feces were obtained by the "wet method" described by Reifenstein, Albright and Wells (17). Dietary, urinary, and fecal nitrogen were done by a macro-Kjeldahl method (18) and plasma protein and non-protein nitrogen by a micro-Kjeldahl method (18). Phosphorus was determined by the method of Fiske and Subbarow (19), calcium according to Fiske and Logan (20), creatinine as described by Peters (21), glucose by the method of Nelson (22), and chloride as described by Van Slyke (23). Uric acid was done by Archibald's modification of the method of Kern and Stransky as described by Forsham and associates (24). Sodium and potassium were determined on a Beckman flame photometer. Sodium determinations were done first and the potassium standards and samples for analysis of potassium brought to the highest concentration of sodium present in the samples. The hematocrit, $\mathrm{CO}_{2}$ content, and $\mathrm{pH}$ of blood were determined by the technique of Shock and Hastings (25). From these data the bicarbonate content of plasma was calculated. Eosinophil counts were done by Forsham's modification of the method of Dungar (24). In the cases of $R$. K. and B. R., urinary steroids were determined in Dr. Dobriner's laboratory.

Formaldehydogenic and 17-ketosteroids were determined after an extraction procedure described by Lieberman and Dobriner (26). The values reported are the sum of the values in the three fractions described in this procedure. Formaldehydogenic steroids were determined on these fractions by a procedure essentially that of Corcoran and Page (27).

On the other subjects $17-$ ketosteroids were done by the method of Callow as modified by Talbot and colleagues $(28,29)$ and formaldehydogenic steroids by the method of Corcoran and Page 1 (27).

Subjects. R. K. was a 31-year-old Negro. Although a childhood disease, presumably poliomyelitis, had resulted in atrophy of some muscle groups in the lower extremities, he did not require artificial support in walking. General health had been excellent and no abnormalities were found on physical examination. Hematological data and the results of urine and stool examination were normal. $C_{I N}$ was $85 \mathrm{cc}$., $C_{P A B}, 397 \mathrm{cc}$. and $\mathrm{Tm}_{\mathrm{G}}$,

1 We are indebted to Dr. F. E. Houghton, Ciba Pharmaceutical Products, Inc., for the supply of desoxycorticosterone used in this method.
$260 \mathrm{mg}$. per minute per 1.73 sq. m.2 The cause of these low values was not apparent. There was no history of renal disease and urinalysis and intravenous and retrograde pyelograms were normal. Following $0.4 \mathrm{mg}$. of adrenaline subcutaneously there was a 64 per cent fall in the concentration of circulating eosinophils. Fifteen days before the start of the present study he completed a period of 12 days of administration of $100 \mathrm{mg}$. of ACTH per day. His response to that agent was normal.

B. R. was a 32-year-old white man admitted because of balanitis due to a beta hemolytic streptococcus. This infection cleared promptly during treatment with sulfadiazine. General health had been good, no abnormalities were found on physical examination, and hematological data and the results of urine and stool analyses were normal. $\mathrm{C}_{\mathrm{IN}}$ was $129 \mathrm{cc}$., $\mathrm{C}_{\mathrm{PAB}}, 896 \mathrm{cc}$., and TmG, 386 mg. per minute per 1.73 sq. m. Following $0.4 \mathrm{mg}$. of adrenaline subcutaneously there was a 54 per cent fall in the concentration of circulating eosinophils. Fifteen days before the start of the present study he completed a period of 12 days of administration of $100 \mathrm{mg}$. of ACTH per day. His response to that agent was normal.

T. B. was a 67-year-old white man whose general health had been excellent. No abnormalities were found on physical examination, urinalysis, and hematological and stool examination. $C_{\mathrm{IN}}$ was $79 \mathrm{cc}$., $\mathrm{T}_{\mathrm{PAB}} 78 \mathrm{mg}$., and Tma $243 \mathrm{mg}$. per minute per 1.73 sq. m. These are normal values for his age group. Following $0.3 \mathrm{mg}$. of adrenaline subcutaneously there was a 47 per cent fall in circulating eosinophils, a normal response for his age group.

G. K., a 42-year-old Negro, whose general health had been good, had a herniorrhaphy a month prior to this study. The only physical abnormality found on examination was a moderately intense short rough apical systolic murmur. Hematological data and the results of stool examination and urinalysis were normal except for the presence of five to 10 leukocytes per high power field in the urinary sediment. Teleoroentgenogram of the chest and electrocardiogram were normal. $C_{\text {IN }}$ was $111 \mathrm{cc}$. and Tma, $341 \mathrm{mg}$. per minute per 1.73 sq. m. There was a 71 per cent fall in eosinophil concentration after $0.3 \mathrm{mg}$. of adrenaline subcutaneously.

Experimental procedure. After a period of equilibration, R. K. and B. R. were studied during a three-day control period, a three-day period of administration of $0.4 \mathrm{mg}$. of adrenaline in saline subcutaneously every four hours, a three-day period of administration of $1 \mathrm{mg}$. of adrenaline in peanut oil intramuscularly every four hours, and a six-day recovery period. After a period of adjustment to the diet, G. K. was studied during a six-day control period, a three-day period of administration of

2 Glomerular filtration rate $\left(C_{I N}\right)$, effective renal plasma flow $\left(C_{P A B}\right)$, maximal tubular excretory rate of sodium p-aminohippurate ( $\left.\mathrm{Tm}_{\mathbf{P A B}}\right)$, and maximal rate of tubular glucose reabsorption $\left(\mathrm{Tm}_{\mathrm{G}}\right)$ were determined by methods described elsewhere (30). 
peanut oil 3 alone intramuscularly every four hours, a second three-day control period, a three-day period of administration of $1 \mathrm{mg}$. of adrenaline in peanut oil intramuscularly every four hours, and a three-day recovery period.

The periods for $T$. B. were the same except that the period of administration of adrenaline in oil lasted six days during the last three of which $1.5 \mathrm{mg}$. of adrenaline was given every four hours, and that the recovery period lasted four days.

The blood pressure, recorded as the mean of seven consecutive readings, and the pulse rate were determined every morning at 7:45 a.m. before the subjects arose. Fasting blood samples were drawn just before the urine collections for the day were completed at 8:00 a.m. Injections of adrenaline were started at 9:00 a.m. Thus, the fasting blood samples were drawn three hours after the 5:00 a.m. injection of adrenaline. In two subjects blood for eosinophil counts was also drawn at 1:00 p.m., four hours after the preceding injection of adrenaline.

The daily urinary excretions of sodium, potassium, chloride, nitrogen, calcium, phosphorus, uric acid, creatinine, glucose, 17-ketosteroids, and formaldehydogenic steroids

${ }^{3}$ Both the adrenaline in peanut oil and the oil alone to serve as a control were supplied through the courtesy of Dr. E. A. Sharpe of Parke, Davis, and Company. The adrenaline was manufactured before this company introduced a process to remove nor-epinephrine. were determined for all subjects and in the cases of R. K. and B. R., fecal sodium, potassium, chloride, nitrogen, calcium, and phosphorus were also estimated. Serum sodium, potassium, chloride, calcium, phosphorus, $\mathrm{pH}$, bicarbonate content, non-protein nitrogen, protein, uric acid, and glucose, and blood eosinophil counts were determined at appropriate intervals.

The daily dietary intakes for G. $K$. and T. B. were water 2800 cc., sodium 91 meq., potassium 89 meq., chloride 84 meq., calcium 269 mg., phosphorus 1228 mg., and nitrogen $16.1 \mathrm{gm}$.; for R. K., water $2800 \mathrm{cc}$., sodium 96 meq., chloride 90 meq., potassium 71 meq., calcium 260 mg., phosphorus $1163 \mathrm{mg}$., and nitrogen $16.0 \mathrm{gm}$.; and for B. R., water 2800 cc., sodium 96 meq., chloride 91 meq., potassium 78 meq., calcium $273 \mathrm{mg}$., phosphorus $1182 \mathrm{mg}$. , and nitrogen $16.1 \mathrm{gm}$.

\section{RESULTS}

The data on functions affected by the administration of adrenaline are recorded in Tables I through IV. In no instance were any alterations in function observed during the administration of peanut oil alone.

On the first day of administration of adrenaline there was in every case a decrease in the concentration of eosinophils in the peripheral blood four

TABLE I

Daily metabolic data* on subject $R . K . \dagger$

\begin{tabular}{|c|c|c|c|c|c|c|c|c|c|c|c|c|c|}
\hline \multirow[b]{2}{*}{ Period } & \multirow[b]{2}{*}{$\begin{array}{l}\text { Wt. of } \\
\text { Pt. }\end{array}$} & \multirow[b]{2}{*}{$\begin{array}{l}\text { Urine } \\
\text { vol. }\end{array}$} & \multicolumn{2}{|c|}{ Sodium } & \multicolumn{2}{|c|}{ Chloride } & \multicolumn{2}{|c|}{ Potassium } & \multirow{2}{*}{$\begin{array}{c}\text { Calcium } \\
\text { Urine }\end{array}$} & \multirow{2}{*}{$\begin{array}{c}\text { Uric acid } \\
\text { Urine }\end{array}$} & \multicolumn{2}{|c|}{ Urinary steroids } & \multirow{2}{*}{$\begin{array}{l}\text { Blood } \\
\text { eooino- } \\
\text { phils }\end{array}$} \\
\hline & & & Urine & Bal. & Urine & Bal. & Urine & Bal. & & & $\begin{array}{l}\text { 17-keto- } \\
\text { steroids }\end{array}$ & $\begin{array}{l}\text { Formalde- } \\
\text { hydogenic } \\
\text { steroids }\end{array}$ & \\
\hline Control & $\begin{array}{c}\text { kg. } \\
68.1 \\
68.0 \\
68.1\end{array}$ & $\begin{array}{c}c c . \\
1990 \\
1470 \\
1700\end{array}$ & $\begin{array}{r}\text { meq. } \\
82 \\
95 \\
84\end{array}$ & $\begin{array}{l}\text { meq. } \\
+5 \\
+8 \\
+3\end{array}$ & $\begin{array}{r}\text { meq. } \\
81 \\
87 \\
81\end{array}$ & $\begin{array}{l}\text { meq. } \\
+2 \\
+4 \\
+2\end{array}$ & $\begin{array}{l}\text { meq. } \\
41 \\
44 \\
44\end{array}$ & $\begin{array}{l}\text { meq. } \\
+2 \\
+1 \\
-1\end{array}$ & $\begin{array}{l}m g . \\
118 \\
124 \\
116\end{array}$ & $\begin{array}{l}8 m . \\
.59 \\
.59 \\
.59\end{array}$ & $\begin{array}{l}m g . \\
16 \\
16 \\
16\end{array}$ & $\begin{array}{l}\text { mg. } \\
0.2 \\
0.2 \\
0.2\end{array}$ & $\begin{array}{c}\text { cells/ } \\
\text { cu. mm. } \\
\frac{67}{67}\end{array}$ \\
\hline $\begin{array}{l}\text { Adrenaline in saline } \\
2.4 \mathrm{mg} . / \mathrm{day}\end{array}$ & $\begin{array}{l}68.2 \\
67.7 \\
68.4\end{array}$ & $\begin{array}{l}1960 \\
1380 \\
1260\end{array}$ & $\begin{array}{r}111 \\
78 \\
68\end{array}$ & $\begin{array}{l}-28 \\
+5 \\
+15\end{array}$ & $\begin{array}{l}85 \\
66 \\
64\end{array}$ & $\begin{array}{l}-4 \\
+15 \\
+17\end{array}$ & $\begin{array}{l}32 \\
28 \\
32\end{array}$ & $\begin{array}{r}+9 \\
+13 \\
+9\end{array}$ & $\begin{array}{l}163 \\
154 \\
151\end{array}$ & $\begin{array}{l}.80 \\
.70 \\
.69\end{array}$ & $\begin{array}{l}15 \\
15 \\
15\end{array}$ & $\begin{array}{l}0.4 \\
0.4 \\
0.4\end{array}$ & $\begin{array}{r}103 \\
55 \\
64\end{array}$ \\
\hline $\begin{array}{l}\text { Adrenaline in oil } \\
6 \mathrm{mg} / \mathrm{day}\end{array}$ & $\begin{array}{l}68.2 \\
67.4 \\
67.2\end{array}$ & $\begin{array}{l}1380 \\
1580 \\
1250\end{array}$ & $\begin{array}{l}115 \\
123 \\
102\end{array}$ & $\begin{array}{l}-32 \\
-40 \\
-19\end{array}$ & $\begin{array}{r}105 \\
112 \\
99\end{array}$ & $\begin{array}{l}-24 \\
-31 \\
-18\end{array}$ & $\begin{array}{l}30 \\
30 \\
40\end{array}$ & $\begin{array}{l}+11 \\
+11 \\
+1\end{array}$ & $\begin{array}{l}193 \\
165 \\
164\end{array}$ & $\begin{array}{l}.92 \\
.86 \\
.69\end{array}$ & $\begin{array}{l}17 \\
17 \\
17\end{array}$ & $\begin{array}{l}0.5 \\
0.5 \\
0.5\end{array}$ & $\begin{array}{l}\mathbf{5 5} \\
\mathbf{5 8} \\
\mathbf{3 3}\end{array}$ \\
\hline Recovery & $\begin{array}{l}67.2 \\
67.6 \\
67.9 \\
68.4 \\
68.4 \\
68.8 \\
68.6\end{array}$ & $\begin{array}{r}1150 \\
960 \\
1200 \\
1420 \\
1160 \\
1620\end{array}$ & $\begin{array}{l}40 \\
11 \\
11 \\
29 \\
46 \\
83\end{array}$ & $\begin{array}{r}+47 \\
+76 \\
+76 \\
+58 \\
+41 \\
+4\end{array}$ & $\begin{array}{l}52 \\
27 \\
30 \\
32 \\
43 \\
68\end{array}$ & $\begin{array}{l}+31 \\
+56 \\
+53 \\
+51 \\
+40 \\
+15\end{array}$ & $\begin{array}{l}52 \\
50 \\
54 \\
44 \\
38 \\
39\end{array}$ & $\begin{array}{r}-12 \\
-10 \\
-14 \\
-4 \\
+2 \\
+1\end{array}$ & $\begin{array}{r}110 \\
82 \\
84 \\
85 \\
86 \\
103\end{array}$ & $\begin{array}{l}.58 \\
.69 \\
.68 \\
.73 \\
.67 \\
.69\end{array}$ & $\begin{array}{l}13 \\
13 \\
13 \\
14 \\
14 \\
14\end{array}$ & $\begin{array}{l}0.6 \\
0.6 \\
0.6 \\
0.7 \\
0.7 \\
0.7\end{array}$ & $\begin{array}{l}\frac{55}{74} \\
\frac{72}{-} \\
\frac{66}{7}\end{array}$ \\
\hline
\end{tabular}

* The balances shown in this and subsequent tables are adjusted for the apparent positive balances of the control period which were of the magnitude usually observed and probably due largely to cutaneous losses. These adjusted balances are the deviations of the daily balances from the average daily balances of the control period.

$\dagger$ Daily intake was: water, 2800 cc., $\mathrm{Na}, 96$ meq., $\mathrm{Cl}, 90$ meq., $\mathrm{K}, 71$ meq., and $\mathrm{Ca}, 260 \mathrm{mg}$. 
TABLE II

Daily metabolic data on subject $B . R^{*}$

\begin{tabular}{|c|c|c|c|c|c|c|c|c|c|c|c|c|c|}
\hline \multirow[b]{2}{*}{ Period } & \multirow{2}{*}{$\begin{array}{c}\text { Wt. of } \\
\text { Pt. }\end{array}$} & \multirow{2}{*}{$\begin{array}{l}\text { Urine } \\
\text { vol. }\end{array}$} & \multicolumn{2}{|c|}{ Sodium } & \multicolumn{2}{|c|}{ Chloride } & \multicolumn{2}{|c|}{ Potassium } & \multirow{2}{*}{$\frac{\text { Calcium }}{\text { Urine }}$} & \multirow{2}{*}{$\begin{array}{c}\text { Uric acid } \\
\text { Urine }\end{array}$} & \multicolumn{2}{|c|}{ Urinary steroids } & \multirow{2}{*}{$\begin{array}{c}\text { Blood } \\
\text { eosino- } \\
\text { phils }\end{array}$} \\
\hline & & & Urine & Bal. & Urine & Bal. & Urine & Bal. & & & $\begin{array}{l}\text { 17-keto- } \\
\text { steroids }\end{array}$ & $\begin{array}{c}\text { Formalde-- } \\
\text { hydogenic } \\
\text { steroids }\end{array}$ & \\
\hline Control & $\begin{array}{c}\text { kg. } \\
50.9 \\
51.0 \\
50.7\end{array}$ & $\begin{array}{c}c c . \\
1680 \\
2140 \\
1670\end{array}$ & $\begin{array}{r}\text { meq. } \\
82 \\
85 \\
74\end{array}$ & $\begin{array}{l}\text { meq. } \\
-2 \\
-5 \\
+6\end{array}$ & $\begin{array}{r}\text { meq. } \\
87 \\
80 \\
73\end{array}$ & $\begin{array}{r}\text { meq. } \\
-7 \\
+\quad 7\end{array}$ & $\begin{array}{c}\text { meq. } \\
54 \\
53 \\
53\end{array}$ & $\begin{array}{r}\text { meq. } \\
-\quad 1 \\
0 \\
0\end{array}$ & $\begin{array}{l}m g . \\
112 \\
120 \\
117\end{array}$ & $\begin{array}{l}g m . \\
.88 \\
.88 \\
.88\end{array}$ & $\begin{array}{l}m g . \\
21 \\
21 \\
21\end{array}$ & $\begin{array}{l}m g . \\
0.1 \\
0.1 \\
0.1\end{array}$ & 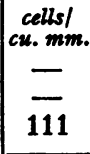 \\
\hline $\begin{array}{l}\text { Adrenaline in saline } \\
2.4 \mathrm{mg} / \mathrm{day}\end{array}$ & $\begin{array}{l}50.9 \\
51.1 \\
51.1\end{array}$ & $\begin{array}{l}1490 \\
1810 \\
1640\end{array}$ & $\begin{array}{l}74 \\
90 \\
98\end{array}$ & $\begin{array}{l}+7 \\
-9 \\
-17\end{array}$ & $\begin{array}{l}61 \\
81 \\
92\end{array}$ & $\begin{array}{l}+19 \\
-1 \\
-12\end{array}$ & $\begin{array}{l}29 \\
37 \\
43\end{array}$ & $\begin{array}{l}+26 \\
+18 \\
+12\end{array}$ & $\begin{array}{l}159 \\
173 \\
162\end{array}$ & $\begin{array}{l}.86 \\
.92 \\
.93\end{array}$ & $\begin{array}{l}22 \\
22 \\
22\end{array}$ & $\begin{array}{l}0.1 \\
0.1 \\
0.1\end{array}$ & $\begin{array}{r}134 \\
70 \\
77\end{array}$ \\
\hline $\begin{array}{l}\text { Adrenaline in oil } \\
6 \mathrm{mg} . / \text { day }\end{array}$ & $\begin{array}{l}51.1 \\
50.6 \\
50.5\end{array}$ & $\begin{array}{l}2230 \\
1890 \\
2320\end{array}$ & $\begin{array}{r}140 \\
97 \\
90\end{array}$ & $\begin{array}{l}-59 \\
-16 \\
-9\end{array}$ & $\begin{array}{r}124 \\
97 \\
92\end{array}$ & $\begin{array}{l}-44 \\
-17 \\
-12\end{array}$ & $\begin{array}{l}48 \\
46 \\
69\end{array}$ & $\begin{array}{l}+7 \\
+9 \\
-14\end{array}$ & $\begin{array}{l}215 \\
174 \\
145\end{array}$ & $\begin{array}{l}.95 \\
.90 \\
.94\end{array}$ & $\begin{array}{l}21 \\
21 \\
21\end{array}$ & $\begin{array}{l}1.1 \\
1.1 \\
1.1\end{array}$ & $\begin{array}{r}106 \\
70 \\
64\end{array}$ \\
\hline Recovery & $\begin{array}{l}50.2 \\
50.6 \\
50.9 \\
51.6 \\
51.8 \\
51.7 \\
51.8\end{array}$ & $\begin{array}{l}1250 \\
1330 \\
1110 \\
1480 \\
1410 \\
1800\end{array}$ & $\begin{array}{r}20 \\
8 \\
22 \\
32 \\
52 \\
57\end{array}$ & $\begin{array}{r}+60 \\
+72 \\
+58 \\
+48 \\
+28 \\
+23\end{array}$ & $\begin{array}{l}45 \\
24 \\
35 \\
36 \\
53 \\
62\end{array}$ & $\begin{array}{r}+35 \\
+56 \\
+45 \\
+44 \\
+27 \\
+18\end{array}$ & $\begin{array}{l}78 \\
54 \\
73 . \\
43 \\
50 \\
50\end{array}$ & $\begin{array}{r}-24 \\
0 \\
-19 \\
+11 \\
+4 \\
+4\end{array}$ & $\begin{array}{r}68 \\
66 \\
88 \\
85 \\
111 \\
111\end{array}$ & $\begin{array}{l}.83 \\
.68 \\
.86 \\
.75 \\
.84 \\
.82\end{array}$ & $\begin{array}{l}19 \\
19 \\
19 \\
20 \\
20 \\
20\end{array}$ & $\begin{array}{l}0.5 \\
0.5 \\
0.5 \\
1.2 \\
1.2 \\
1.2\end{array}$ & $\begin{array}{l}\frac{70}{72} \\
\frac{61}{\overline{66}}\end{array}$ \\
\hline
\end{tabular}

* Daily intake was: water, 2800 cc., Na, 96 meq., Cl, 91 meq., K, 78 meq., and $\mathrm{Ca}, 273$ mg.

TABLE III

Daily metabolic data on subject $G . K$.* $^{*}$

\begin{tabular}{|c|c|c|c|c|c|c|c|c|c|c|c|c|c|}
\hline \multirow[b]{2}{*}{ Period } & \multirow[b]{2}{*}{$\begin{array}{l}\text { Wt. of } \\
\text { Pt. }\end{array}$} & \multirow{2}{*}{$\begin{array}{l}\text { Urine } \\
\text { vol. }\end{array}$} & \multicolumn{2}{|c|}{ Sodium } & \multicolumn{2}{|c|}{ Chloride } & \multicolumn{2}{|c|}{ Potassium } & \multirow{2}{*}{$\begin{array}{c}\text { Calcium } \\
\text { Urine }\end{array}$} & \multirow{2}{*}{$\begin{array}{c}\text { Uric acid } \\
\text { Urine }\end{array}$} & \multicolumn{2}{|c|}{ Urinary steroids } & \multirow{2}{*}{$\begin{array}{l}\text { Blood } \\
\text { eosino- } \\
\text { phils }\end{array}$} \\
\hline & & & Urine & Bal. & Urine & Bal. & Urine & Bal. & & & $\begin{array}{l}\text { 17-keto- } \\
\text { steroids }\end{array}$ & $\begin{array}{c}\text { Formalde- } \\
\text { hydogenic } \\
\text { steroids }\end{array}$ & \\
\hline Control & $\begin{array}{c}\text { kg. } \\
61.2 \\
61.6 \\
61.6 \\
61.6 \\
61.6 \\
61.5\end{array}$ & $\begin{array}{c}c c . \\
1860 \\
2060 \\
2200 \\
2070 \\
2330 \\
2100\end{array}$ & $\begin{array}{r}\text { meq. } \\
68 \\
77 \\
82 \\
86 \\
93 \\
87\end{array}$ & $\begin{array}{r}\text { meq. } \\
+14 \\
+5 \\
0 \\
-4 \\
-11 \\
-5\end{array}$ & $\begin{array}{r}\text { meq. } \\
62 \\
70 \\
75 \\
84 \\
79 \\
77\end{array}$ & $\begin{array}{r}\text { meq. } \\
+12 \\
+4 \\
-1 \\
-10 \\
-5 \\
-3\end{array}$ & $\begin{array}{c}\text { meq. } \\
60 \\
68 \\
66 \\
72 \\
65 \\
62\end{array}$ & $\begin{array}{r}\text { meq. } \\
+5 \\
-3 \\
-1 \\
-7 \\
0\end{array}$ & $\begin{array}{l}m g . \\
39 \\
35 \\
35 \\
40 \\
40 \\
33\end{array}$ & $\begin{array}{l}g m . \\
.86 \\
.86 \\
.79 \\
.79 \\
.76 \\
.74\end{array}$ & $\begin{array}{r}m g . \\
9 \\
9 \\
9 \\
10 \\
10 \\
10\end{array}$ & $\begin{array}{l}m g . \\
1.0 \\
1.0 \\
1.0 \\
1.0 \\
1.0 \\
1.0\end{array}$ & $\begin{array}{c}\text { cellsl } \\
\text { cu. mm. } \\
- \\
- \\
- \\
- \\
\overline{465}\end{array}$ \\
\hline Oil & $\begin{array}{l}61.1 \\
61.5 \\
61.4\end{array}$ & $\begin{array}{l}1720 \\
2290 \\
1830\end{array}$ & $\begin{array}{l}93 \\
72 \\
70\end{array}$ & $\begin{array}{l}-11 \\
+10 \\
+12\end{array}$ & $\begin{array}{l}83 \\
75 \\
66\end{array}$ & $\begin{array}{l}-9 \\
\overline{1} \quad 1 \\
+8\end{array}$ & $\begin{array}{l}71 \\
74 \\
71\end{array}$ & $\begin{array}{l}-6 \\
=9 \\
-6\end{array}$ & $\begin{array}{l}41 \\
42 \\
44\end{array}$ & $\begin{array}{l}.76 \\
.73 \\
.71\end{array}$ & $\begin{array}{l}10 \\
10 \\
10\end{array}$ & $\begin{array}{l}0.8 \\
0.8 \\
0.8\end{array}$ & $\begin{array}{l}417 \\
456 \\
498\end{array}$ \\
\hline Control & $\begin{array}{l}61.4 \\
61.4 \\
61.2\end{array}$ & $\begin{array}{l}1880 \\
2070 \\
2030\end{array}$ & $\begin{array}{l}79 \\
81 \\
81\end{array}$ & $\begin{array}{l}+3 \\
+1 \\
+1\end{array}$ & $\begin{array}{l}69 \\
76 \\
73\end{array}$ & $\begin{array}{l}+5 \\
-2 \\
+1\end{array}$ & $\begin{array}{l}65 \\
73 \\
67\end{array}$ & $\begin{array}{r}0 \\
-\quad 8 \\
-\quad 2\end{array}$ & $\begin{array}{l}37 \\
48 \\
51\end{array}$ & $\begin{array}{l}.74 \\
.78 \\
.75\end{array}$ & $\begin{array}{l}9 \\
9 \\
9\end{array}$ & $\begin{array}{l}0.7 \\
0.7 \\
0.7\end{array}$ & $\frac{470}{-}$ \\
\hline $\begin{array}{l}\text { Adrenaline in oil } \\
6 \text { mg./day }\end{array}$ & $\begin{array}{l}61.5 \\
61.0 \\
60.9\end{array}$ & $\begin{array}{l}2380 \\
2450 \\
2250\end{array}$ & $\begin{array}{l}107 \\
137 \\
132\end{array}$ & $\begin{array}{l}-25 \\
-55 \\
-50\end{array}$ & $\begin{array}{r}66 \\
110 \\
132\end{array}$ & $\begin{array}{l}+8 \\
-36 \\
-58\end{array}$ & $\begin{array}{l}47 \\
65 \\
72\end{array}$ & $\begin{array}{r}+18 \\
0 \\
-\quad 7\end{array}$ & $\begin{array}{l}89 \\
70 \\
67\end{array}$ & $\begin{array}{l}.85 \\
.82 \\
.80\end{array}$ & $\begin{array}{l}6 \\
6 \\
6\end{array}$ & $\begin{array}{l}0.9 \\
0.9 \\
0.9\end{array}$ & $\begin{array}{l}454 \\
419 \\
600\end{array}$ \\
\hline Recovery & $\begin{array}{l}60.4 \\
60.7 \\
60.7 \\
61.2\end{array}$ & $\begin{array}{l}1760 \\
1750 \\
1750\end{array}$ & $\begin{array}{l}87 \\
47 \\
40\end{array}$ & $\begin{array}{r}-5 \\
+35 \\
+42\end{array}$ & $\begin{array}{r}103 \\
53 \\
42\end{array}$ & $\begin{array}{l}-29 \\
+21 \\
+32\end{array}$ & $\begin{array}{l}94 \\
70 \\
73\end{array}$ & $\begin{array}{l}-29 \\
=5 \\
-8\end{array}$ & $\begin{array}{l}40 \\
45 \\
43\end{array}$ & $\begin{array}{l}.73 \\
.76 \\
.71\end{array}$ & $\begin{array}{l}8 \\
8 \\
8\end{array}$ & $\begin{array}{l}0.8 \\
0.8 \\
0.8\end{array}$ & $\begin{array}{l}537 \\
355 \\
294 \\
412\end{array}$ \\
\hline
\end{tabular}

* Daily intake was: water, 2800 cc., Na, 91 meq., Cl, 84 meq., K, 89 meq., and Ca, 269 mg. 
TABLE IV

Daily metabolic data on subject $T . B .^{*}$

\begin{tabular}{|c|c|c|c|c|c|c|c|c|c|c|c|c|c|}
\hline \multirow[b]{2}{*}{ Period } & \multirow[b]{2}{*}{$\begin{array}{c}\text { Wt. of } \\
\mathrm{Pt} .\end{array}$} & \multirow[b]{2}{*}{$\begin{array}{l}\text { Urine } \\
\text { vol. }\end{array}$} & \multicolumn{2}{|c|}{ Sodium } & \multicolumn{2}{|c|}{ Chloride } & \multicolumn{2}{|c|}{ Potassium } & \multirow{2}{*}{$\begin{array}{l}\text { Calcium } \\
\text { Urine }\end{array}$} & \multirow{2}{*}{$\begin{array}{c}\text { Uric acid } \\
\text { Urine }\end{array}$} & \multicolumn{2}{|c|}{ Urinary steroids } & \multirow[b]{2}{*}{$\begin{array}{c}\text { Blood } \\
\text { eosino- } \\
\text { phils }\end{array}$} \\
\hline & & & Urine & Bal. & Urine & Bal. & Urine & Bal. & & & $\begin{array}{l}\text { 17-keto- } \\
\text { steroids }\end{array}$ & $\begin{array}{l}\text { Formalde- } \\
\text { hydogenic } \\
\text { steroids }\end{array}$ & \\
\hline Control & $\begin{array}{c}\text { kg. } \\
80.0 \\
80.0 \\
80.1 \\
80.1 \\
80.1 \\
80.1\end{array}$ & $\begin{array}{c}c c . \\
1770 \\
1710 \\
1960 \\
1790 \\
2210 \\
1810\end{array}$ & $\begin{array}{c}\text { meq. } \\
69 \\
64 \\
84 \\
83 \\
96 \\
72\end{array}$ & $\begin{array}{r}\text { meq. } \\
+9 \\
+14 \\
-6 \\
-5 \\
-18 \\
+6\end{array}$ & $\begin{array}{l}\text { meq. } \\
64 \\
59 \\
70 \\
73 \\
80 \\
62\end{array}$ & $\begin{array}{r}\text { meq. } \\
+4 \\
+9 \\
-2 \\
-5 \\
-12 \\
+6\end{array}$ & $\begin{array}{c}\text { meq. } \\
68 \\
62 \\
72 \\
71 \\
77 \\
67\end{array}$ & $\begin{array}{l}\text { meq. } \\
+1 \\
+7 \\
+3 \\
-2 \\
-8 \\
+2\end{array}$ & $\begin{array}{r}m g . \\
100 \\
88 \\
104 \\
80 \\
91 \\
103\end{array}$ & $\begin{array}{l}g m . \\
.56 \\
.80 \\
.87 \\
.84 \\
.83 \\
.83\end{array}$ & $\begin{array}{c}m g . \\
5 \\
5 \\
5 \\
6 \\
6 \\
6\end{array}$ & $\begin{array}{l}m g . \\
1.0 \\
1.0 \\
1.0 \\
0.7 \\
0.7 \\
0.7\end{array}$ & $\begin{array}{c}\text { cellsl } \\
\text { cu. mm. } \\
- \\
- \\
- \\
- \\
\overline{409}\end{array}$ \\
\hline Oil & $\begin{array}{l}80.0 \\
80.0 \\
80.0\end{array}$ & $\begin{array}{l}2020 \\
1930 \\
1530\end{array}$ & $\begin{array}{l}75 \\
84 \\
70\end{array}$ & $\begin{array}{l}+3 \\
\pm 6 \\
+8\end{array}$ & $\begin{array}{l}68 \\
76 \\
66\end{array}$ & $\begin{array}{r}0 \\
-8 \\
+\quad 2\end{array}$ & $\begin{array}{l}70 \\
72 \\
70\end{array}$ & $\begin{array}{l}-1 \\
=3 \\
-1\end{array}$ & $\begin{array}{r}108 \\
109 \\
98\end{array}$ & $\begin{array}{l}.85 \\
.84 \\
.82\end{array}$ & $\begin{array}{l}6 \\
6 \\
6\end{array}$ & $\begin{array}{l}1.0 \\
1.0 \\
1.0\end{array}$ & $\begin{array}{l}310 \\
346 \\
483\end{array}$ \\
\hline Control & $\begin{array}{l}80.1 \\
80.0 \\
79.9\end{array}$ & $\begin{array}{l}2220 \\
1620 \\
1550\end{array}$ & $\begin{array}{l}91 \\
80 \\
64\end{array}$ & $\begin{array}{l}-13 \\
-2 \\
+14\end{array}$ & $\begin{array}{l}80 \\
73 \\
66\end{array}$ & $\begin{array}{l}-12 \\
-5 \\
+2\end{array}$ & $\begin{array}{l}78 \\
73 \\
69\end{array}$ & $\begin{array}{r}-9 \\
-4 \\
0\end{array}$ & $\begin{array}{r}116 \\
95 \\
100\end{array}$ & $\begin{array}{l}.81 \\
.75 \\
.79\end{array}$ & $\begin{array}{l}6 \\
6 \\
6\end{array}$ & $\begin{array}{l}0.7 \\
0.7 \\
0.7\end{array}$ & $\frac{450}{-}$ \\
\hline $\begin{array}{l}\text { Adrenaline in oil } \\
6 \mathrm{mg} . / \text { day }\end{array}$ & $\begin{array}{l}79.9 \\
79.4 \\
79.4\end{array}$ & $\begin{array}{l}2040 \\
1770 \\
1560\end{array}$ & $\begin{array}{l}88 \\
98 \\
87\end{array}$ & $\begin{array}{l}-10 \\
-20 \\
-9\end{array}$ & $\begin{array}{l}68 \\
74 \\
77\end{array}$ & $\begin{array}{r}0 \\
-\quad 6 \\
-\quad 9\end{array}$ & $\begin{array}{l}49 \\
46 \\
52\end{array}$ & $\begin{array}{l}+20 \\
+23 \\
+17\end{array}$ & $\begin{array}{l}140 \\
136 \\
129\end{array}$ & $\begin{array}{l}.85 \\
.95 \\
.78\end{array}$ & $\begin{array}{l}6 \\
6 \\
6\end{array}$ & $\begin{array}{l}0.8 \\
0.8 \\
0.8\end{array}$ & $\begin{array}{l}340 \\
230 \\
334\end{array}$ \\
\hline $\begin{array}{l}\text { Adrenaline in oil } \\
9 \mathrm{mg} . / \text { day }\end{array}$ & $\begin{array}{l}79.7 \\
79.4 \\
79.3\end{array}$ & $\begin{array}{l}1220 \\
1490 \\
1680\end{array}$ & $\begin{array}{l}82 \\
85 \\
90\end{array}$ & $\begin{array}{l}-4 \\
-7 \\
-12\end{array}$ & $\begin{array}{l}71 \\
78 \\
78\end{array}$ & $\begin{array}{l}-3 \\
-10 \\
-10\end{array}$ & $\begin{array}{l}59 \\
64 \\
72\end{array}$ & $\begin{array}{l}+10 \\
+5 \\
-3\end{array}$ & $\begin{array}{l}136 \\
139 \\
125\end{array}$ & $\begin{array}{r}1.23 \\
.90 \\
.71\end{array}$ & $\begin{array}{l}6 \\
6 \\
6\end{array}$ & $\begin{array}{l}0.8 \\
0.8 \\
0.8\end{array}$ & $\begin{array}{l}537 \\
640 \\
577\end{array}$ \\
\hline Recovery & $\begin{array}{l}79.4 \\
79.6 \\
79.6 \\
79.6 \\
79.9\end{array}$ & $\begin{array}{l}1040 \\
1300 \\
1170 \\
1270\end{array}$ & $\begin{array}{l}42 \\
44 \\
61 \\
82\end{array}$ & $\begin{array}{l}+36 \\
+34 \\
+17 \\
-\quad 4\end{array}$ & $\begin{array}{l}58 \\
55 \\
65 \\
81\end{array}$ & $\begin{array}{l}+10 \\
+13 \\
+3 \\
-13\end{array}$ & $\begin{array}{l}86 \\
85 \\
80 \\
69\end{array}$ & $\begin{array}{r}-17 \\
-16 \\
-11 \\
0\end{array}$ & $\begin{array}{r}75 \\
93 \\
98 \\
111\end{array}$ & $\begin{array}{l}.65 \\
.67 \\
.83 \\
.74\end{array}$ & $\begin{array}{l}5 \\
5 \\
5 \\
5\end{array}$ & $\begin{array}{l}0.8 \\
0.8 \\
0.8 \\
0.8\end{array}$ & $\begin{array}{l}415 \\
593 \\
583 \\
394\end{array}$ \\
\hline
\end{tabular}

* Daily intake was: water, 2800 cc., Na, 91 meq., Cl, 84 meq., K, 89 meq., and $\mathrm{Ca}, 269 \mathrm{mg}$.

hours after the first injection of adrenaline. In subjects $R$. K. and B. R., the level of circulating eosinophils at 8:00 a.m., three hours after the preceding injection of adrenaline, was moderately decreased throughout the entire period of administration of adrenaline. After the withdrawal of adrenaline, the eosinophil concentration rose toward the control values in R. K., but not in B. R. In the other two subjects, although there was a fall in the concentration of eosinophils following the first injection of adrenaline, the values during the latter part of the period were actually higher than the control values. The 1:00 p.m. levels of circulating eosinophils determined only in $\mathrm{R}$. $\mathrm{K}$. and B. R. approximated the 8:00 a.m. values.

During the administration of adrenaline there was no change in 17-ketosteroid excretion. During and following adrenaline, R. K. and B. R. showed slightly increased formaldehydogenic steroid excretion. Even if these changes are regarded as significant they indicate no more than a mild degree of adrenocortical stimulation. The other two subjects showed no change in formaldehydogenic steroid excretion.

The urinary uric acid was slightly increased during the entire period of administration of adrenaline and returned to the control values after withdrawal of adrenaline. There was no consistent change in plasma uric acid.

The average increase in plasma glucose during the administration of adrenaline was $15 \mathrm{mg}$. per $100 \mathrm{cc}$. During this period the average increase in urinary glucose excretion was $1.2 \mathrm{gm}$. per day.

There were no consistent changes in the fecal excretions of the elements studied in the two cases in which stool analyses were done. Adjusted balances for sodium, potassium, and chloride are recorded in Tables I through IV. Unadjusted balances during the control period were positive. These positive balances were of the magnitude 
usually observed in control periods and probably due to cutaneous losses. The average daily positive balance of the control period was assumed to represent cutaneous loss and was subtracted from the observed balance for each day. In the two cases in which fecal determinations were not made, fecal excretions were also assumed to be constant. The adjustment in these two cases includes fecal excretion as well as cutaneous loss. Balances derived in this way are referred to as adjusted balances.

No consistent change in the urinary excretion of sodium and chloride occurred during the administration of adrenaline in saline. During the administration of adrenaline in oil, there was an increase in the urinary excretion and concentration of these ions. The subjects were thus in negative sodium and chloride balance during the administration of adrenaline in oil. Subsequently the urinary excretions and concentrations of sodium and chloride fell, and the subjects retained sodium and chloride. There were no consistent changes in the serum concentrations of sodium and chloride.

The changes in the urinary excretion of potassium were in the opposite direction to those of sodium and chloride. There was a decrease in the urinary excretion and concentration of potassium during the administration of adrenaline whether in saline or oil. During the recovery period the urinary excretion and concentration rose. Thus, there were positive balances of potassium during the administration of adrenaline followed by compensatory negative balances in the recovery period. There were no consistent changes in the serum potassium levels.

The urinary calcium excretion and concentration were increased during the entire period of administration of adrenaline and fell to values below the control levels after withdrawal of adrenaline.

There were no consistent changes in the urinary excretion of nitrogen and phosphorus.

The changes in urinary volume were small. In the case of B. R. and G. K., there was an increase in urinary volume during the administration of adrenaline and a diminution in urinary volume in the recovery period. R. K. and T. B. showed no definite change in urinary volume. Because of variations in extrarenal losses of water, changes of this magnitude are of little value in estimating wa- ter balance. However, during adrenaline administration all the subjects lost weight which they regained in the recovery period. The losses and subsequent gains were for R. K., 1.0 and $1.4 \mathrm{~kg}$., for B. R., 0.9 and $1.6 \mathrm{~kg}$., for T. B., 0.5 and $0.5 \mathrm{~kg}$., and for G. K., 1.1 and $0.8 \mathrm{~kg}$. These changes in weight may have been due to changes in body water. If so, then all subjects may be said to have had a larger urinary volume than they would have had with the same extrarenal loss of water if adrenaline had not been given.

The plasma protein concentration increased slightly in every case during the administration of adrenaline in oil. The average increase was 0.4 gm. per $100 \mathrm{cc}$. There were no consistent changes in creatinine excretion, urinary $\mathrm{pH}$, the hematocrit, nor in the serum non-protein nitrogen, phosphorus, bicarbonate, or $\mathrm{pH}$.

The average increase in the 8:00 a.m. pulse rate during the administration of adrenaline in oil was three beats per minute, and in the pulse rate an hour after the 9:00 a.m. injection, three beats per minute over the control value an hour after the administration of oil alone. The average changes in the 8:00 a.m. systolic and diastolic blood pressures were +2 and $-2 \mathrm{~mm}$. of mercury respectively, and in the systolic and diastolic pressures an hour after the 9:00 a.m. injection of adrenaline in oil, +12 and $0 \mathrm{~mm}$. of mercury over the control values an hour after the administration of oil alone.

\section{DISCUSSION}

The only evidence suggestive of increased adrenocortical activity during the administration of adrenaline was the decrease in the level of circulating eosinophils in B. R. and R. K., and the small changes in the formaldehydogenic steroid excretion in these two subjects. The other subjects showed no changes suggestive of altered adrenocortical activity. It is apparent that adrenaline in the time-dosage schedule used in this study is no more than a very weak chronic stimulus to adrenocortical activity.

The changes in the excretion of electrolytes during the administration of adrenaline indicate that renal function was modified by some mechanism other than adrenocortical stimulation. In view of this, the increased urinary excretion of uric acid cannot be considered a valid measure of adrenocor- 
tical activity since this may have been due to renal effects not caused by an adrenocortical hormone.

During the administration of adrenaline in oil there was increased renal excretion of sodium, chloride, and calcium, retention of potassium, and loss of weight. The failure of adrenaline in saline to produce all these changes is presumably due to the smaller dose of epinephrine employed or to its more transitory action. The changes in weight suggest that there was greater renal excretion of water than would have occurred with the same extrarenal loss of water if adrenaline had not been given.

Diuresis of water and chloride has been observed in acute experiments in animals given relatively small amounts of adrenaline (12-16). Although the effect of adrenaline on the renal excretion of sodium, calcium, and potassium was not studied in the animal experiments, it appears probable that the same processes were affected in those experiments as in the one reported here. There is no direct evidence as to the mechanism of the alterations in renal excretion of electrolytes and water, though naturally it has been suggested that they are due to a direct effect of adrenaline on the kidney, altering either glomerular filtration or tubular function (15). An alteration in tubular function is compatible with our data and suggested by the fact that the changes in renal excretion of electrolytes were not all in the same direction, potassium excretion being diminished while sodium, chloride, and calcium excretion were increased. It is also compatible with the observation of Chasis and associates (31) that the subcutaneous injection of $1 \mathrm{mg}$. of adrenaline does not alter glomerular filtration rate in man. The conditions of their experiments did not permit the study of the effect of adrenaline on urinary volume.

The concept that the effect of adrenaline on renal excretion is a direct effect of adrenaline on the kidney rather than the result of changes produced elsewhere in the body is supported by the observation of Richards and Plant (32) that perfusion of the kidney in situ produces diuresis and the observation of Winton (33) that adrenaline produces diuresis in the heart-lung-kidney preparation.

Although the diuresis observed in animal experimentation has been generally attributed to the epinephrine content of adrenaline, Horres, Ever- sole and Rock have recently presented evidence that it is caused by nor-epinephrine and not by epinephrine (16).

\section{SUMMARY AND CONCLUSIONS}

Certain metabolic effects of the chronic administration of adrenal medullary hormones to men are reported.

Adrenal medullary hormones in the time-dose schedule employed are no more than a very weak chronic stimulus to adrenocortical activity.

Under the conditions of this study, the chronic administration of adrenal medullary hormones caused increased urinary excretion of sodium, chloride, calcium, and uric acid, and diminished urinary excretion of potassium.

\section{ACKNOWLEDGMENTS}

We are indebted to Dr. Konrad Dobriner in whose laboratory at the Sloan-Kettering Institute certain of the steroid determinations were done; to Dr. Roger K. McDonald and Dr. John H. Miller for renal function determinations; to Mr. Marvin J. Yiengst for serum bicarbonate and $\mathrm{pH}$ determinations; and to Eloise Bright, Mary Jane Ford, Stewart Humphreys, James Laster, John Melvin, Janis Parsons, Mildred Salchunas, Patricia Skinner, and Millard Starnes for technical assistance.

\section{REFERENCES}

1. Babes, V., and Jonesco, V., Etudes sur la diminution de la graisse surrénale dans des états pathologiques. Compt. rend. Soc. de biol., 1908, 65, 267.

2. Vogt, M., Observations on some conditions affecting rate of hormone output of the suprarenal cortex. J. Physiol., 1944, 103, 317.

3. Vogt, M., The effect of chronic administration of adrenalin on the suprarenal cortex and the comparison of this effect with that of hexoestrol. J. Physiol., 1945, 104, 60.

4. Long, C. N. H., and Fry, E. G., Effect of epinephrine on adrenal cholesterol and ascorbic acid. Proc. Soc. Exper. Biol. \& Med., 1945, 59, 67.

5. Gershberg, H., Fry, E. G., Brobeck, J. R., and Long, C. N. H., The role of epinephrine in the secretion of the adrenal cortex. Yale J. Biol. \& Med., 1950, 23, 32.

6. Recant, L., Hume, D. M., Forsham, P. H., and Thorn, G. W., Studies on the effect of epinephrine on the pituitary-adrenocortical system. J. Clin. Endocrinol., 1950, 10, 187.

7. Bliss, E. L., Rubin, S., and Gilbert, T., The effect of adrenalin on adrenal cortical function. J. Clin. Endocrinol., 1951, 11, 46. 
8. Guest, C. M., Kammerer, W. H., Cecil, R. L., and Berson, S. A., Epinephrine, pregnenolone and testosterone in the treatment of rheumatoid arthritis. J. A. M. A., 1950, 143, 338.

9. Goldenberg, M., Faber, M., Alston, E. J., and Chargaff, E. C., Evidence for the occurrence of norepinephrine in the adrenal medulla. Science, 1949, 109, 534.

10. Pellegrino, P. C., Morris, G. M., and Trubowitz, S., Eosinophil response to epinephrine and nor-epinephrine. Proc. Soc. Exper. Biol. \& Med., 1950, 74, 330.

11. Humphreys, R. J., and Raab, W., Response of circulating eosinophils to nor-epinephrine, epinephrine and emotional stress in humans. Proc. Soc. Exper. Biol. \& Med., 1950, 74, 302.

12. Samson, P. C., and Jacobs, H. R. D., Some chemical effects from constant intravenous epinephrine injections in dogs. Am. J. Physiol., 1932, 99, 433.

13. Toth, L. A., The effects of epinephrine on urine excretion in dogs. Am. J. Physiol., 1937, 119, 140.

14. Gaunt, R., Liling, M., and Cordsen, M., Adrenal medulla in water diuresis and water intoxication. Endocrinology, 1945, 37, 136.

15. Hays, H. W., and Mathieson, D. R., Studies on water intoxication in adrenalectomized rats and the influence of desoxycorticosterone acetate (DCA) and epinephrine in water diuresis. Endocrinology, 1945, 37, 147.

16. Horres, A. D., Eversole, W. J., and Rock, M., Adrenal medullary hormones in water diuresis. Proc. Soc. Exper. Biol. \& Med., 1950, 75, 58.

17. Reifenstein, E. C., Jr., Albright, F., and Wells, S. L., The accumulation, interpretation, and presentation of data pertaining to metabolic balances, notably those of calcium, phosphorus, and nitrogen. J. Clin. Endocrinol., 1945, 5, 367.

18. Hiller, A., Plazin, J., and Van Slyke, D. D., A study of conditions for Kjeldahl determination of nitrogen in proteins. Description of methods with mercury as catalyst, and titrimetric and gasometric measurements of the ammonia formed. J. Biol. Chem., 1948, 176, 1401.

19. Fiske, C. H., and Subbarow, Y., The colorimetric determination of phosphorus. J. Biol. Chem., 1925, 66, 375 .

20. Fiske, C. H., and Logan, M. A., The determination of calcium by alkalimetric titration. II. The precipitation of calcium in the presence of magnesium, phosphate, and sulfate, with application to the analysis of urine. J. Biol. Chem., 1931, 93, 211.

21. Peters, J. H., The determination of creatinine and creatine in blood and urine with the photoelectric colorimeter. J. Biol. Chem., 1942, 146, 179.

22. Nelson, N., A photometric adaptation of the Somogyi method for the determination of glucose. J. Biol. Chem., 1944, 153, 375.

23. Peters, J. P., and Van Slyke, D. D., Quantitative Clinical Chemistry. Vol. II, Methods. Williams \& Wilkins Co., Baltimore, 1932, p. 833.

24. Forsham, P. H., Thorn, G. W., Prunty, F. T. G., and Hills, A. G., Clinical studies with pituitary adrenocorticotropin. J. Clin. Endocrinol., 1948, 8, 15.

25. Shock, N. W., and Hastings, A. B., Studies of the acid-base balance of the blood. I. A microtechnique for the determination of the acid-base balance of the blood. J. Biol. Chem., 1934, 104, 565.

26. Lieberman, S., and Dobriner, K., Steroid excretion in health and disease; chemical aspects, in: Recent Progress in Hormone Research. Academic Press, Inc., N. Y., 1948, Vol. 3, p. 71.

27. Corcoran, A. C., and Page, I. H., Methods for the chemical determination of corticosteroids in urine and plasma. J. Lab. \& Clin. Med., 1948, 33, 1326.

28. Talbot, N. B., Butler, A. M., and MacLachlan, E., The colorimetric assay of total, $\alpha$-, and $\beta-17$-ketosteroids in extracts of human urine. J. Biol. Chem., 1940, 132, 595.

29. Talbot, N. B., Berman, R. A., and MacLachlan, E. A.; Elimination of errors in the colorimetric assay of neutral urinary 17 -ketosteroids by means of a color correction equation. J. Biol. Chem., 1942, 143, 211.

30. Miller, J. H., McDonald, R. K., and Shock, N. W., The effect of bacitracin on renal function. J. Clin. Invest., 1950, 29, 389.

31. Chasis, H., Ranges, H. A., Goldring, W., and Smith, H. W., The control of renal blood flow and glomerular filtration in normal man. J. Clin. Invest., 1938, 17, 683.

32. Richards, A. N., and Plant, O. H., The action of minute doses of adrenalin and pituitrin on the kidney. Am. J. Physiol., 1922, 59, 191.

33. Winton, F. R., The control of the glomerular pressure by vascular changes within the isolated mammalian kidney, demonstrated by the actions of adrenaline. J. Physiol., 1931, 73, 151. 